\title{
Impact of Deep N-well Implantation on Substrate Noise Coupling and RF Transistor Performance for Systems-on-a-Chip Integration
}

\author{
K. W. Chew, J. Zhang, K. Shao, W. B. Loh and S-. F. Chu \\ Chartered Semiconductor Manufacturing Ltd, Mixed-Signal/RFCMOS Process Integration Group \\ Woodlands Industrial Park D, Singapore 738406 \\ Email: chewkw@charteredsemi.com
}

\begin{abstract}
This paper reviews the merits of incorporating deep $n$-well implantation in state-of-the-art CMOS technologies to address mixed-mode coupling in integrated circuits. The deep n-well architecture, coupled with novel body biasing techniques and the use of $p+$ guard ring, have resulted in a maximum of $35 \mathrm{~dB}$ reduction in substrate noise at $100 \mathrm{MHz}$. Furthermore the deep n-well implantation does not impact the $d c, a c$, rf and noise performance of the multi-fingered transistor, hence allowing model consistency with the standard well multi-fingered transistor.
\end{abstract}

\section{Introduction}

The aggressive scaling of CMOS technologies has enabled the realization of monolithic systems that integrate high-speed digital circuits with highperformance analog circuits [1], [2], and the ultimate goal includes the integration of wireless RF circuitries as well. In such mixed-mode systems, substrate noise coupling has been identified as a major problem [3], [4], [5].

There are three main mechanisms of noise injection into the substrate. Firstly digital transition switching is the primary cause of substrate noise injection in mixedsignal integrated circuits. These transients can couple into the sensitive analog circuits, thereby limiting their analog precision. The fast switching transients produced in digital circuits have most of their spectral content in the higher harmonics of the clock. Hence for a $100 \mathrm{MHz}$ clock, substrate noise coupling above $1 \mathrm{GHz}$ becomes significant. This problem is further compounded by the demands for higher clock rates and greater analog precision for each new generation of products. Consequently this hastens the need for a viable solution. Secondly the inductances associated with the metal connections, bond wires and package lead frame combine with the on-chip capacitances between the power rail and ground to produce ringing of the powersupply voltage. This phenomenon is commonly known as "ground bounce" and it can propagate throughout the substrate via a digital ground that is connected to the substrate [6], [7].
Thirdly with the reduction of device feature sizes, substrate currents due to impact-ionization have gained prominence [8]. However these unwanted currents in the substrate could be minimized by fine-tuning the lightlydoped drain (LDD) implant dose and/or the use of thick gate oxide transistor with a higher supply voltage and a longer channel length.

Hence in order to realize single-chip analog/digital/RF systems that are efficient both in terms of die area and development time, designers have used circuit design, layout styles such as the use of $p+$ guard rings, and careful floor planning [9] to address this issue. This paper discusses the merits and issues of incorporating a deep n-well implant as a process solution to the substrate noise issue in systems-on-a-chip (SoC) design.

\section{Device structure, fabrication and measurements}

Figure 1 illustrates the annotated cross-sectional view of a n-channel transistor enclosed within the deep n-well structure. Note that there are three body or bulk contacts made available by the deep n-well architecture.

The deep n-well process flow adopts the conventional logic CMOS front-end-of-line processing steps that begins with STI formation followed by n-well formation; p-well formation; channel implants; gate insulator and gate electrode formation; pocket implant; LDD implant; nitride spacer formation; source/drain implant; source/drain annealing and finally salicidation. The deep n-well is formed by inserting a high-energy ion implantation step before n-well formation. In this work, deep n-well implant splits consisting of phosphorus doses at $1 \times 10^{13} \mathrm{~cm}^{-2}, 2 \times 10^{13} \mathrm{~cm}^{-2}$ and $3 \times 10^{13} \mathrm{~cm}^{-2}$ have been performed with an implant energy of $900 \mathrm{KeV}$. This implant energy has been optimized in an earlier experiment (not mentioned here) to achieve good noise isolation by completely surrounding the p-well region for junction isolation, yet causing minimal disturbances to the transistor dc characteristics.

Figure 2 illustrates the SIMS profiles for the deep nwell implant dose of $2 \times 10^{13} \mathrm{~cm}^{-2}$ at $900 \mathrm{KeV}$ and the pwell within it. The results indicate that the deep n-well and p-well depths are approximately $2 \mu \mathrm{m}$ and $1.2 \mu \mathrm{m}$ respectively.

Three generations of devices from the $0.13 \mu \mathrm{m}, 0.18$ 
$\mu \mathrm{m}$ and $0.25 \mu \mathrm{m}$ nodes have been fabricated on p-type bulk wafers with resistivites of 6-9 $\Omega-\mathrm{cm}$ and subsequently have been characterized.

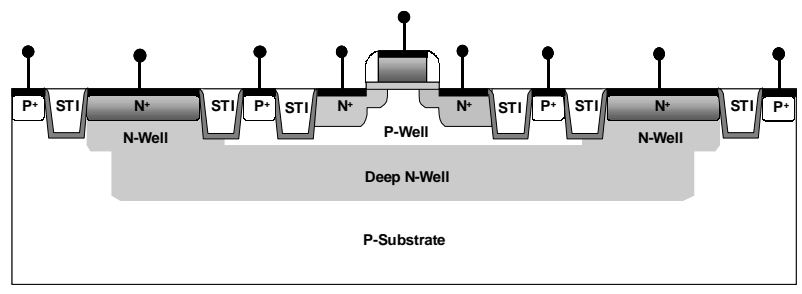

Figure 1. Annotated cross-sectional view of an nchannel transistor within the deep n-well architecture. Note that there are 3 body contacts available for biasing.

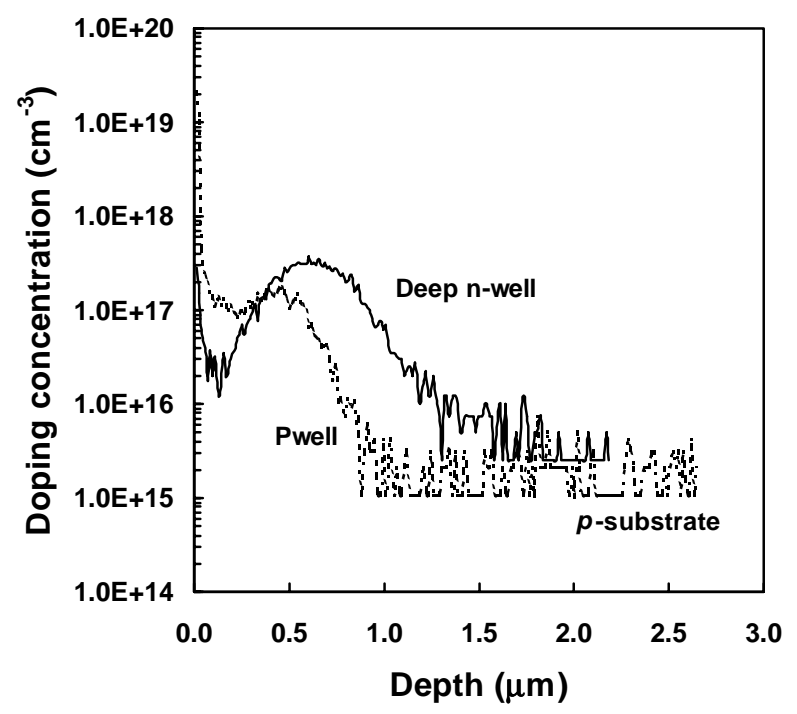

Figure 2. Deep n-well and p-well secondary ion mass spectroscopy (SIMS) profile obtained with a deep n-well implant dose of $2 \times 10^{13} \mathrm{~cm}^{-2}$ at 900 $\mathrm{KeV}$.

\section{Effect on substrate noise coupling}

Each test structure consists of either a $p+$ or $n+$ noise generator, with or without deep n-well, at one terminal and a $p+$ ohmic contact on the receiving terminal. Some structures include a $p+$ guard ring placed $2 \mu \mathrm{m}$ away from the n-well sinker for additional protection. Figure 3 shows the schematic of a typical substrate coupling test structure. Note that the legends in subsequent plots will refer to the labels shown in figure 3 .

Figure 4 shows the $\mathrm{S} 21$ isolation for $p+$ noise generators with $\mathrm{G}$ to $\mathrm{S}$ spacing of $280 \mu \mathrm{m}$ and deep nwell implant dose of $1 \times 10^{13} \mathrm{~cm}^{-2}$ at $900 \mathrm{KeV}$. It reveals that a "floating" deep n-well provides $20 \mathrm{~dB}$ of isolation at $100 \mathrm{MHz}$, as compared with the $p+$ noise generator without deep n-well.

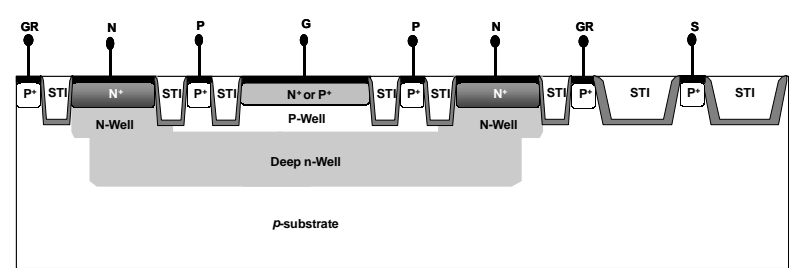

Figure 3. Annotated cross-sectional view of a typical diode-type substrate coupling test structure. $G$ denotes the noise generator, $P$ denotes the $\mathrm{p}$-well pickup, $\mathrm{N}$ denotes the $\mathrm{n}$-well pickup, GR denotes the $p+$ guard ring and $S$ denotes the $p+$ sensor.



Figure 4. Effect of different body biasing techniques on the substrate noise transmission versus frequency for $p+$ noise generators, with $G$ to $S$ spacing of $280 \mu \mathrm{m}$ and deep n-well implant dose of $1 \times 10^{13} \mathrm{~cm}^{-2}$ at $900 \mathrm{KeV}$. DNW stands for deep n-well.

By grounding the deep n-well and $p+$ guard ring, an additional of approximately $15 \mathrm{~dB}$ of isolation can be achieved. The high-pass forward transmission characteristics shown by the noise coupling structures with deep n-well enclosure is expected owning to the formation of resistance-capacitance (RC) networks between the area and periphery junction capacitances in series with the $p$-substrate resistance. Placing a grounded $p+$ guard ring around an injector is known to be an effective substrate noise suppression technique [9].

Figure 5 illustrates the S21 isolation of 3 different protection schemes for $n+$ noise generators, with $\mathrm{G}$ to $\mathrm{S}$ spacing of $280 \mu \mathrm{m}$ and deep n-well implant dose of $1 \times 10^{13} \mathrm{~cm}^{-2}$ at $900 \mathrm{KeV}$. It shows that by connecting the deep n-well and $\mathrm{p}$-well to Vdd, and grounding the $p+$ guard ring, approximately $74 \mathrm{~dB}$ of noise isolation can be achieved. This is because the equipotential p-well and n-well acts like a large n-type sinker. 


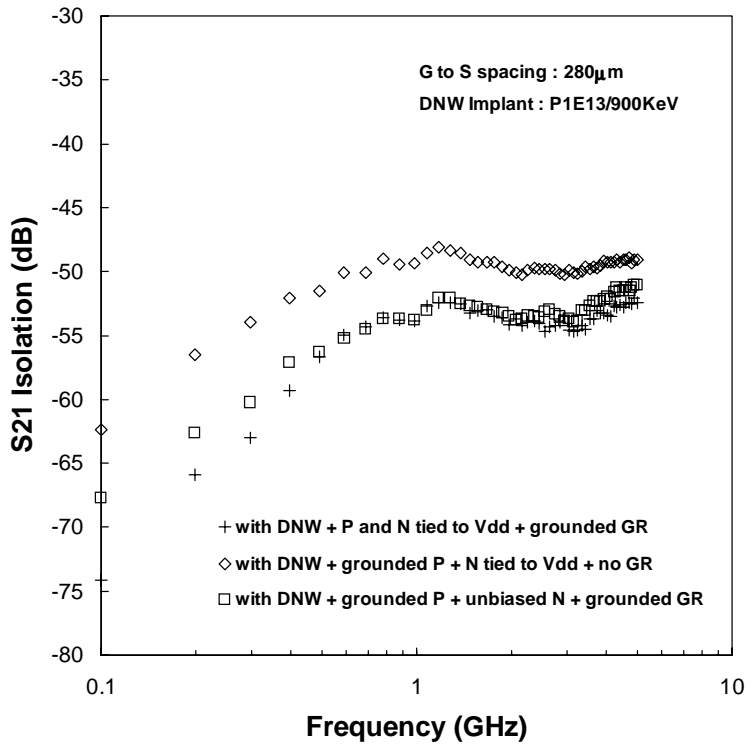

Figure 5. Effect of different body biasing techniques on the substrate noise transmission versus frequency for $n+$ noise generators, with $G$ to $S$ spacing of $280 \mu \mathrm{m}$ and deep n-well implant dose of $1 \times 10^{13} \mathrm{~cm}^{-2}$ at $900 \mathrm{KeV}$.

Figure 6 shows the S21 isolation of different protection schemes for $n+$ noise generators, but with a tighter $\mathrm{G}$ to $\mathrm{S}$ spacing of $50 \mu \mathrm{m}$ and a slightly higher deep n-well implant dose of $2 \times 10^{13} \mathrm{~cm}^{-2}$ at $900 \mathrm{KeV}$. The data for $p+$ noise generator $(\diamond)$ without any deep nwell has been added for comparison. The plot shows that the $p+$ noise generator is the dominant source of substrate noise, as compared to the $n+$ noise generator.

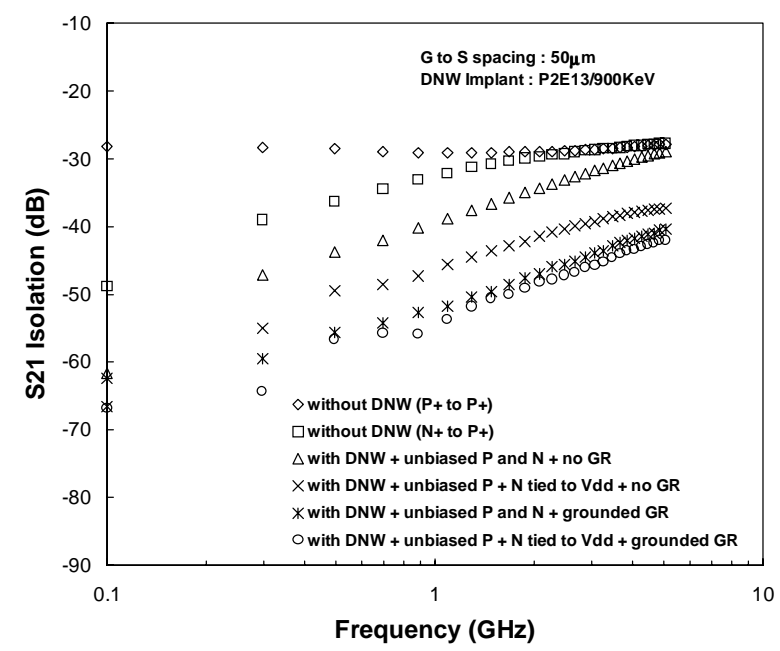

Figure 6. Effect of different body biasing techniques on the substrate noise transmission versus frequency for $n+$ noise generators, with $G$ to $S$ spacing of $50 \mu \mathrm{m}$ and deep n-well implant dose of $2 \times 10^{13} \mathrm{~cm}^{-2}$ at $900 \mathrm{KeV}$.
Figure 6 shows that by connecting the deep n-well to Vdd, and grounding the $p+$ guard ring, approximately 67 $\mathrm{dB}$ of noise isolation can be achieved. As compared to the $n+$ noise generator without deep $\mathrm{n}$-well, there is a suppression of about $18 \mathrm{~dB}$ of substrate noise at 100 MHz.

Figure 7 illustrates the effect of deep n-well implant dose for a $p+$ noise generator with grounded deep $\mathrm{n}$-well and $p+$ guard ring. The results indicate no significant differences in isolation at $200 \mathrm{MHz}$ but a $2 \mathrm{~dB}$ improvement for doses of $2 \times 10^{13} \mathrm{~cm}^{-2}$ and $3 \times 10^{13} \mathrm{~cm}^{-2}$ above $400 \mathrm{MHz}$.

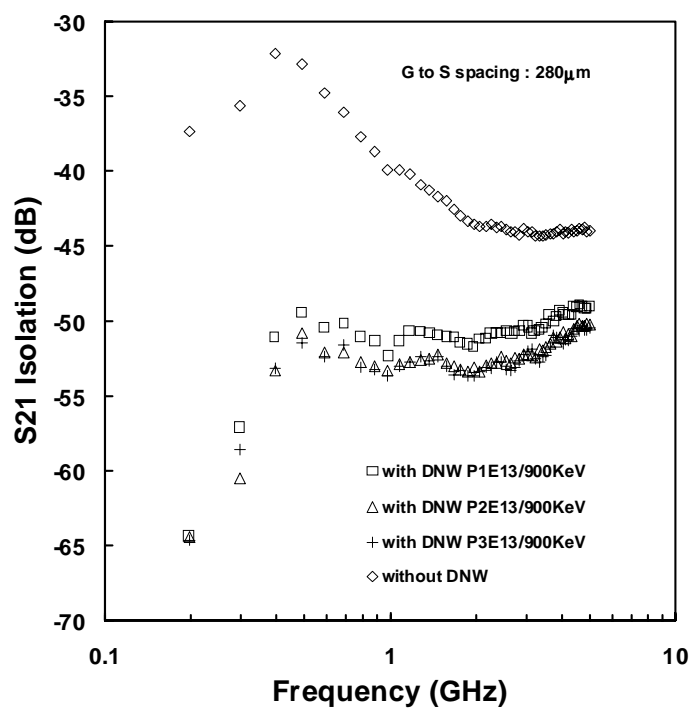

Figure 7. Effect of varying the deep n-well implant dose on the substrate noise transmission versus frequency for a $p+$ noise generator with grounded deep n-well and $p+$ guard ring and with $G$ to $S$ spacing of $280 \mu \mathrm{m}$.

\section{Effect on RF transistor performance}

Figure 8 indicates that the deep n-well process produces minimal discrepancies on the dc characteristics. Figure 9 shows the impact of deep n-well implant on the ac capacitances of the RF transistor. Figure 10 illustrates that the RF transistor high-frequency characteristics with and without deep n-well remains consistent. This implies that the incorporation of deep n-well will not have any impact on the RF transistor model. Figure 11 indicates no significant change in the minimum noise figure $\mathrm{NF}_{\min }$ with deep n-well implant. Figure 12 illustrates the flicker noise current spectral density of the RF transistor with and without deep n-well implant. A slight improvement in flicker noise is observed for the transistor with a deep $\mathrm{n}$-well implant. The deep n-well implant dose used in the comparison is $1 \times 10^{13} \mathrm{~cm}^{-2}$ at $900 \mathrm{KeV}$. 


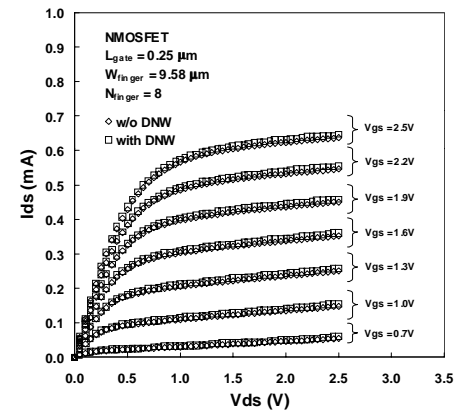

Figure 8. Impact of deep n-well on the dc characteristics of the RF transistor.

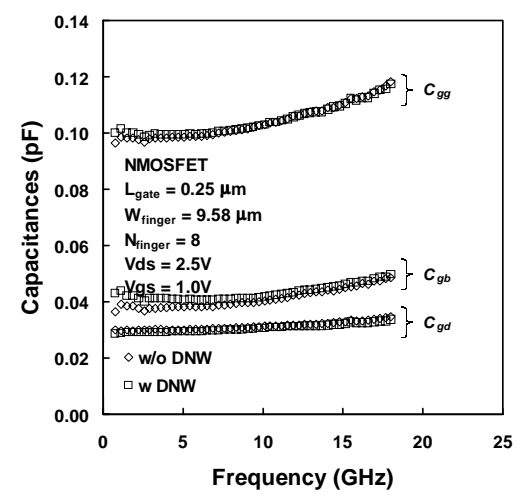

Figure 9. Effect of varying the deep n-well implant dose on $C_{g g}, C_{g b}$ and $C_{g d}$ of the RF transistor. It has been assumed that $C_{g d}$ and $C_{g s}$ are approximately equivalent.

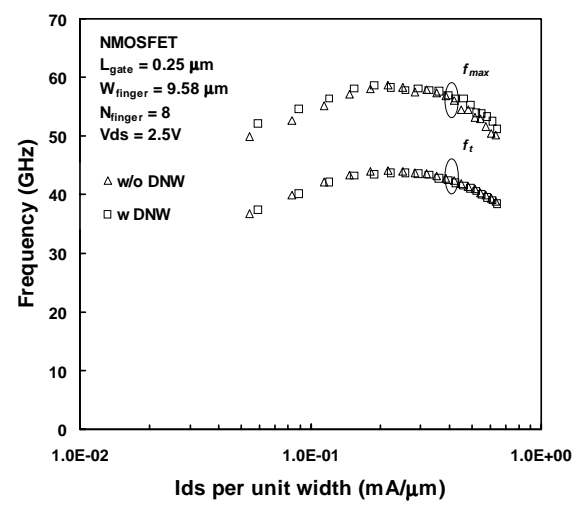

Figure 10. Effect of deep n-well implant on the $t_{t}$ and $f_{\max }$ of the RF transistor versus Ids/ $\mu \mathrm{m}$-width.

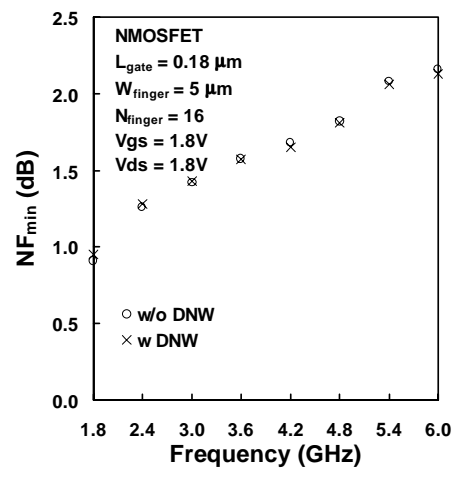

Figure 11. Impact of deep n-well implant on the minimum noise figure $\mathrm{NF}_{\text {min }}$ of the $\mathrm{RF}$ transistor.

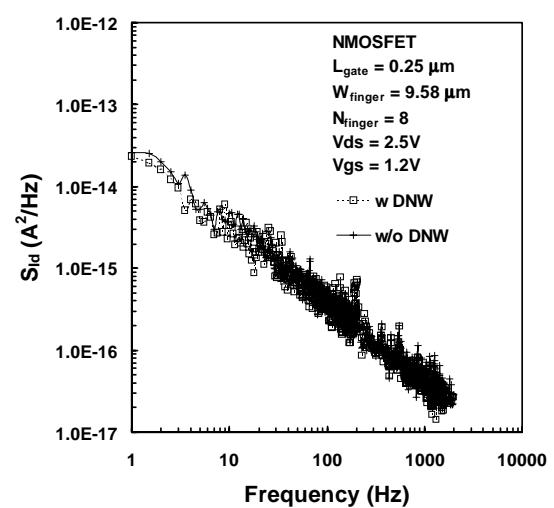

Figure 12. Effect of deep n-well implant on the flicker noise current spectral density $S_{l d}$ of the RF transistor.

\section{Conclusion}

The deep n-well architecture, coupled with different body biasing techniques, has resulted in a maximum of $35 \mathrm{~dB}$ improvement in substrate noise isolation at 100 MHz. The deep n-well process does not impact the $\mathrm{dc}$, $\mathrm{rf}$ and noise performance of the multi-fingered CMOS transistor.

\section{References}

[1] T. J. Schmerbeck et al., "A $27 \mathrm{MHz}$ mixed A/D magnetic recording channel DSP using partial response signaling with maximum likelihood detection," in ISSCC Dig. Tech. Papers, 1991.

[2] S. Takeuchi et al., "A 30-MHz mixed analog/digital signal processor," IEEE J. Solid-State Circuits, vol. 25, pp. 1458-1463, Dec. 1990.

[3] D. K. Su et al., "Experimental results and modeling techniques for substrate noise in mixed-signal integrated circuits," IEEE J. Solid-State Circuits, vol. 28, pp. 420-430, Apr. 1993.

[4] N. K. Verghese et al., "Computer-aided design considerations for mixed-signal coupling in RF integrated circuits," IEEE J. Solid-State Circuits, vol. 33, no. 3, pp. 314323, Mar. 1998.

[5] M. V. Heijningen, et al., "Analysis and experimental verification of digital substrate noise generation for epi-type substrates," IEEE J. Solid-State Circuits, vol. 35, no. 7, pp. 1002-1008, July 2000.

[6] T. Gabara, "Reduced ground bounce and improved latch-up suppression through substrate conduction," IEEE $J$. Solid-State Circuits, vol. 23, pp. 1224-1232, Oct. 1998.

[7] R. Senthinathan et al., "Simultaneous switching ground noise calculation for packaged CMOS devices," IEEE J. Solid-State Circuits, vol. 26, pp. 1724-1728, Nov. 1991.

[8] J. Briaire et al., "Substrate injection and crosstalk in CMOS circuits," in Proc. IEEE Custom Integrated Circuits Conf., 1999, pp. 483-486.

[9] Jerry Twomey, "Noise reduction is crucial to mixedsignal ASIC design success (part I)," Electron Design, pp. 123131, Oct. 30, 2000. 